\title{
High-Reynolds-number turbulence in complex fluids
}

\author{
D. Kulmatova ${ }^{1}$, D. Bonn ${ }^{2}$ and H. Kellay ${ }^{3}$ \\ ${ }^{1}$ Laboratoire de Physique Statistique, Ecole Normale Supérieure - 24 rue Lhomond, 75005 Paris, France, EU \\ ${ }^{2}$ Van der Waals-Zeeman Institute, University of Amsterdam - Science Park 904, 1098 XH Amsterdam, \\ The Netherlands, EU \\ ${ }^{3}$ U. Bordeaux 1, Laboratoire Ondes et Matière d'Aquitaine, UMR 5798 CNRS - 351 cours de la Libération \\ 33405 Talence, France, EU
}

PACS 47.27. Jv - High-Reynolds-number turbulence

PACS $47.57 . \mathrm{Ng}$ - Polymers and polymer solutions

\begin{abstract}
We here examine the structure of turbulence in the case of a complex fluid made up of water and surfactants. This fluid has the particular property of shear thickening when driven at shear rates above a certain threshold. Through a study of the spectral properties and the structure function scalings, important differences arise with respect to the reference case, i.e., water. The surfactant solution shows strong intermittency at small scales. The large scales are, on the other hand, free of intermittency. While this transition is observed in the structure function scalings, no sign of this transition is seen in the power spectrum of velocity fluctuations which shows a single scaling range. The strongly intermittent small-scale region, despite the scaling of the power spectrum, exhibits properties reminiscent of the near-dissipative range.
\end{abstract}

Turbulence in fluids is widespread in both natural and industrial settings. Since the early works of Richardson and Kolmogorov, many features of turbulent flows in simple fluids are known even if some are not fully understood $[1,2]$. That $3 \mathrm{D}$ turbulence in simple fluids is governed by a direct cascade of energy from the large scales to the small scales giving rise to a well-defined energy density spectrum across the scales is now fully accepted. That a closer look at finer details of such a state through the so-called structure functions of the velocity field yields anomalies is another known fact. Such anomalies can be phenomenologically explained starting with the work of Kolmogorov himself [2]. Nonetheless, this anomalous behavior is believed to be at the heart of a full understanding of this ubiquitous state. In natural and industrial settings, however, the fluids or liquids are far from simple: rivers transport particles and sediment, oceans are host to a variety of mesoscopic and microscopic entities, and industrial fluids are usually mixtures of complicated molecules. It has been known for a long time now that some properties of turbulence, at least at the macroscopic scale, can be influenced by the addition of minute amounts of polymers, fibers or surfactants for that matter $[3,4]$. Despite the large interest in such modifications and their possible use for drag reduction in pipes, the structure of the turbulence is still unclear. Recent work (and less recent work) did show modifications of the energy density spectrum [5] and the second-order structure function [6] at scales near the dissipative range and beyond, raising additional questions as to how such modifications may occur and what their role may be.

We here reexamine the issue of the modification of the structure of turbulence, using a dilute surfactant solution as the complex fluid, through the possible modifications of the scaling behavior of the energy density spectrum and the velocity structure functions. We use the so-called von Karman cell in which the turbulence is generated in a large cylindrical tank by two counterrotating discs. As the disks are equipped with baffles, the energy dissipation is independent of the viscosity, which is a prerequisite for having interial turbulence [7]. At the same time, if drag reducting agents are added to such a flow, no drag reduction is observed since the latter is due to boundary-layer effects that are suppressed by using the baffles [8]. This puts us in a confortable situation to invesigate the effect of complex fluids on the structure of the turbulence itself. Because the overall dissipation does not change upon addition of surfactants, one can compare flows with and without the complex fluid at the same energy dissipation. Perhaps our most important result is the presence of strong intermittency at small scales for the surfactant solution but practically no intermittency 
at large scales indicating that the structure of the fluid itself maybe playing a strong role. This difference between the small-scale and the large-scale properties is however absent in the energy density spectrum which shows the same scaling law for the two ranges. We argue that the nature of the fluid plays a role in setting the anomalies with respect to the pure Kolmogorov case opening the possibility of probing these anomalies through the fine tuning of the fluid properties.

We use an experimental apparatus allowing for a turbulent flow between two counterrotating baffled disks. Such a set up has been extensively used for the examination of turbulence properties in simple fluids as well as in drag reducing complex fluids [7-10]. This apparatus, due to the manner in which the turbulence is created (i.e., the baffles instead of a flat and smooth surface), does not present drag reduction. What we examine is therefore the modification of the turbulence properties in the bulk of the solution. We use Laser Doppler Velocimetry (LDV), which produces temporal traces of the velocity of micrometer-sized seed particles in solution and measured at a fixed location in the cell. Our reference state is water and the complex fluid used is a dilute aquaeous solution. The Reynolds numbers examined are defined as $R^{2} \Omega / \nu$ and range from 5000 to 100000 ( $R$ is the radius of the disk, $\Omega$ is the angular rotation, and $\nu$ is the kinematic viscosity of the fluids used). The dimensions of the cell are a radius of $7 \mathrm{~cm}$ and a height of $20 \mathrm{~cm}$. For the measurements to be presented here, estimates of the Taylor microscale yield values near $0.5 \mathrm{~mm}$ with the associated Reynolds number taking values near 200. The standard deviation of the velocity fluctuations can be as high as $50 \mathrm{~cm} / \mathrm{s}$ yielding high turbulent intensities that can reach $50 \%$. This standard deviation takes smaller values for the surfactant solution with values near $20 \mathrm{~cm} / \mathrm{s}$. The two disks are equipped with four baffles of height $4 \mathrm{~cm}$. The two disks are mounted on two separate motors that can rotate at different rotation frequencies controlled by two current supplies. Measurements of the power injection vs. the rotation frequency indicate that this power increases as the third power of the rotation frequency as expected and is independent of the fluid used and therefore of the viscosity confirming previous observations [8]. The cell was then filled with approximately $2.5 \mathrm{l}$ of the desired solution. We have used surfactant solutions of $0.5 \%$ CTAB and $0.1 \% \mathrm{NaSal}$ (by weight). In this concentration range, the solution is composed of worm-like micelles in the dilute regime [10]. The rheological characterization of these solutions was carried out using a rheometer. This surfactant solution is shear thickening as has been shown before [10]. The viscosity of the solution is near that of water for shear rates below a threshold value (which varies between 20 and $100 \mathrm{~s}^{-1}$ ). Above this shear rate, the viscosity increases abruptly by over a factor 5 and then decreases slightly as the shear rate increases [10]. The rheological properties of this complex fluid are therefore very different from the often used polymer solutions which generally show shear thinning behavior.
For comparison, we have also carried out experiments using a long chain polymer solution, PEO of $4 \cdot 10^{6}$ molecular weight at $50 \mathrm{ppm}$ by weight.

We now turn to the description of the turbulence properties. The first feature we have examined is the energy density spectrum obtained from the time series of the longitudinal velocity fluctuations. The LDV probe was positioned slightly below the center of the cell so as to have a non-zero mean flow velocity. The probe can be positioned at different distances from the wall and we chose to do our measurements between 5 and $10 \mathrm{~mm}$ from the wall. These considerations are for practical reasons: in order to increase the count rate of the LDV, a sufficient amount of particles has to be dispersed in solution. At such concentrations, measurements deep into the solution become complicated due to multiple scattering by the particles in the bulk fluid. While we checked that variations of this distance between a few millimeters from the wall and at least $1 \mathrm{~cm}$ from the wall did not change the major features of the results, we cannot confirm that wall effects are totally absent. However, an estimate of the viscous sublayer width gives $0.5 \mathrm{~mm}$ leading us to conclude that our measurements are at least outside this layer. Other considerations arise: in a recent paper, flow reversals were observed [11]. We do not know how these flow reversals affect the results but runs with practically no reversals were also analyzed with no major changes of the main results.

In fig. 1 we show the energy density spectra obtained for the reference solution and for the polymer solution. At this level, the difference between the polymer solution and the reference solution seems absent. We have recast the frequency into a wave number by using the frozen turbulence assumption when representing the power spectra of the velocity fluctuations. The reference soltution (water for a rotation frequency of $5 \mathrm{~Hz}$ ) shows a range in scales where a power law decrease is observed (fig. 1(a)). Recall that these are one-dimensional energy density spectra obtained from a time series of the velocity along the flow direction. The exponent extracted from this power law decrease is near -1.46 . In the case where the frozen turbulence assumption is valid, we expect an exponent of $-5 / 3$ which is characteristic of Kolmogorov scaling for 3D fluid turbulence. While the value we obtain seems close to the expected value, it is nonetheless smaller. Previous work in a similar cell also measured exponents smaller than $-5 / 3[9]$. Such a feature is probably due to the proximity of the wall and possibly to a failure of the Taylor hypothesis when the intensity of the turbulence is strong, which is the case here. Nonetheless, the spectrum shows scaling and the exponent is not far from the expected one. In addition to this scaling regime, the power spectrum seems to decrease more steeply for scales below about $0.3 \mathrm{~cm}$ (as marked by an arrow in fig. 1(a)). The polymer solution, fig. 1(c), shows a similar behavior: a scaling regime with a roughly similar exponent as water along with a steeper decrease at smaller scales below $0.5 \mathrm{~cm}$ (arrow in fig. 1(c)). 

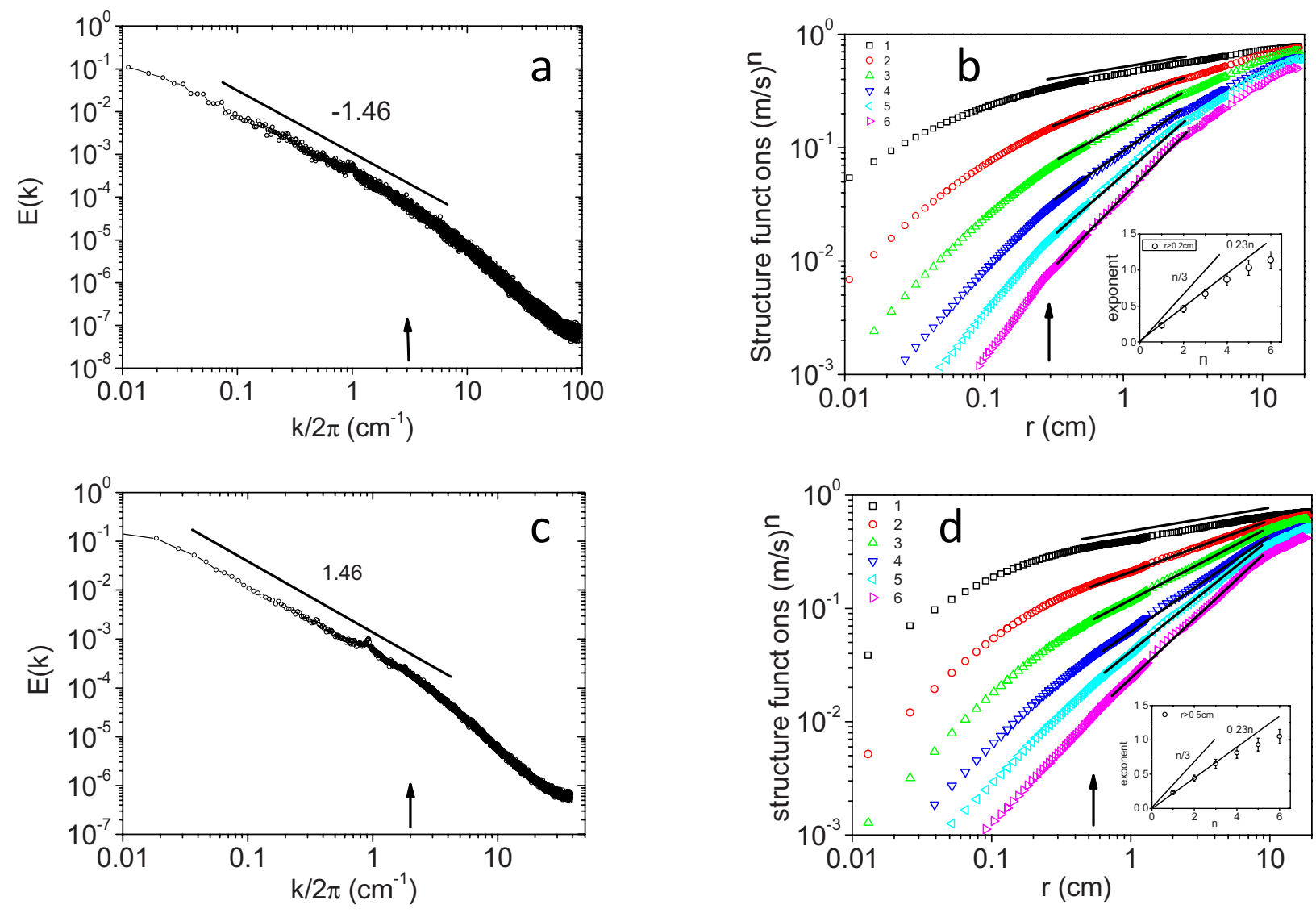

Fig. 1: (Colour on-line) Spectra and velocity structure functions. (a) and (b): water at a rotation frequency of $5 \mathrm{~Hz}$. (c) and (d): polymer solution at $5 \mathrm{~Hz}$. The arrows indicate the approximate transition scale to a steeper spectrum. The insets in (b) and (d) show the variation of the structure function exponents vs. order. The lines indicate the expected Kolmogorov scaling ( $n / 3)$ and the variation found here for the low-order moments.

There are no marked differences between the polymer solution and water.

The surfactant solution also shows power law scaling for the energy density spectrum with a higher exponent near -1.7 as seen in fig. 2(a) and (c) which seems consistent with a Kolmogorov scaling. Apart from differences in the exponents measured, another feature comes out of these spectra: while the water spectrum and the polymer solution spectrum show a steeper decrease at small scales below $0.3 \mathrm{~cm}$ and $0.5 \mathrm{~cm}$, respectively, no such decrease is observed for the surfactant solution up to scales of $0.01 \mathrm{~cm}$. While for the water and the polymer cases, a signature of a near-dissipative range (the dissipative scale is estimated to be 100 micrometers) is a probable cause for the steepening of the spectrum at small scales, no such signature appears in the spectra of the surfactant solution. This is true for both frequencies examined as shown in fig. 2(a) and (c). The peak in the spectra at large scales corresponds to the passage of the baffles; this peak is barely visible in the spectra for water and polymer solutions as the turbulence intensity in this case is higher than for the surfactant solution.

Let us now take a look at the structure functions of the velocity using the LDV scheme and the obtained time traces. These functions are defined as $S_{n}(\tau)=\delta u^{n}(\tau)=$ $\left\langle(u(t+\tau)-u(t))^{n}\right\rangle$, where $u$ is the fluctuating part of the longitudinal velocity in the mean flow direction. Again, the use of the frozen turbulence assumption allows to convert time scales to length scales. For water and the polymer solution, these functions are shown in fig. 1(b) and (d). About a decade of power law scaling is observed, corresponding to the scaling range of the power spectrum: the scaling range for the water case is between roughly 0.3 and $4 \mathrm{~cm}$ in good agreement with the scaling of the energy density spectrum which shows scaling for scales larger than $0.3 \mathrm{~cm}$. The scaling range for the polymer solution is also in agreement with that of the power spectrum and extends roughly between $0.5 \mathrm{~cm}$ and $7 \mathrm{~cm}$. A quantity of interest is the exponent of these power laws and its variation with the order $n$ of the moment. Indeed, and for three-dimensional turbulence, the exponents $\xi_{n}$ characterizing the scaling law for each order $n$ is predicted to be $n / 3$. This rule is displayed by several experiments up to $n=4$ beyond which the exponents vary more slowly [2]. This anomaly is attributed to intermittency of the fluctuations leading to changes in the functional form of the probability density functions of velocity differences as the scale changes. Our results for water and the polymer 

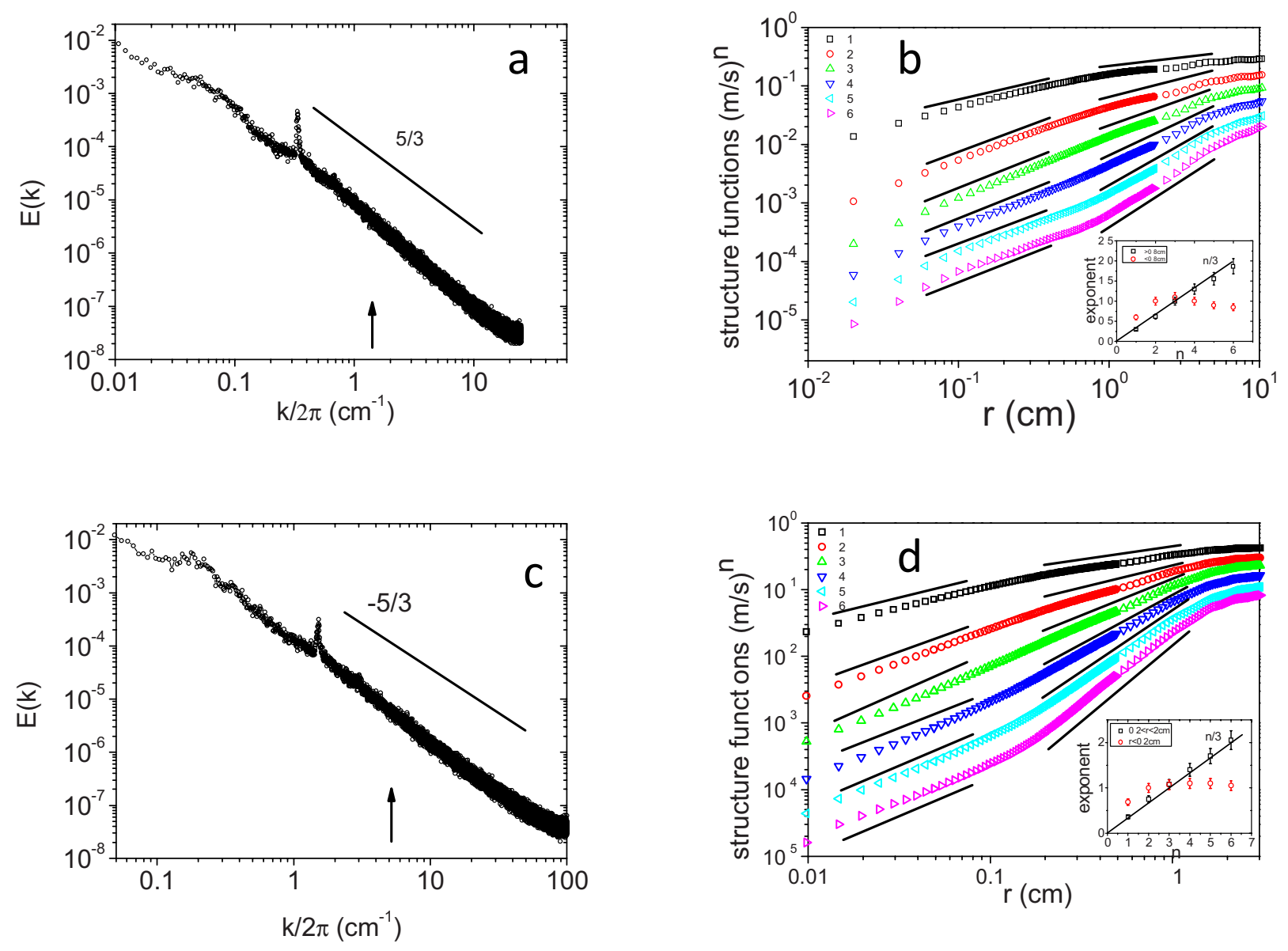

Fig. 2: (Colour on-line) Spectra and velocity structure functions. (a) and (b): CTAB solutions at a frequency of $4 \mathrm{~Hz}$. (c) and (d): CTAB solution at a frequency of $5 \mathrm{~Hz}$. The arrows indicate the scale at which the structure functions show a transition to a more intermittent scaling range. Note that the spectrum in the surfactant case shows no particular transition at this scale. The structure functions were shifted vertically for better visibility: the downward shift is by factors of $1.25,2,4,8$, and 20 for the second to the sixth order, respectively. The peak in the spectrum of the surfactant solution corresponds to 4 times the driving frequency and is indicative of the passage of the baffles; this peak is also present in the water and polymer spectra but its amplitude is much smaller due to the higher turbulent intensity in these fluids as compared to the surfactant solution. The exact significance of this peak and its relation to the scale of energy injection is unclear to us as the distance between two baffles in the outer periphery of the disk is nearly $10 \mathrm{~cm}$ while the position of the peak using the Taylor hypothesis varies from run to run. The insets in (b) and (d) show the variation of the structure function exponents (for the two regimes) vs. order. The lines indicate the expected Kolmogorov scaling $(n / 3)$.

solution show good agreement with the expected result: a roughly linear increase of $\xi_{n}$ (see inset of fig. 1(b) and (d)). For higher moments, the exponent deviates as it is smaller than expected. This behavior is now well established for 3D turbulent systems and our results reproduce such a phenomenology despite the fact that our spectral exponent is not exactly $-5 / 3$. In fact, the increase of the structure function exponents, up to order 4 , can be mimicked using $0.23 n$ instead of $n / 3$ in agreement with the smaller value of the exponent of the velocity power spectrum. The exponent for the second-order structure function is related to the spectral exponent through $\xi_{2}=\alpha-1$, where $\alpha$ is the spectral exponent. This leads to $\xi_{n}=n \xi_{2} / 2=n(\alpha-1) / 2$. For $\alpha=1.46, \quad \xi_{n}=0.23 n$ in good agreement with our results. At scales smaller than $0.3 \mathrm{~cm}$ for water and $0.5 \mathrm{~cm}$ for the polymer solution, the structure functions seem to decrease more steeply in agreement with the steeper decrease of the power spectrum. Here again, the differences between the polymer solution and the water case are very minor: the amplitude of the structure functions for the polymer case at small scales are however smaller than for water, as noted in previous work [6].

For the surfactant solution, power law scaling is also observed (see fig. 2(b) and (d)), but two different ranges seem to exist contrary to the power spectrum where no particular feature was observed as the scaling range seemed to extend down to the smallest scales examined. The first scaling range for the surfactant solution is observed for scales between $0.8 \mathrm{~cm}$ and $8 \mathrm{~cm}$ for the $4 \mathrm{~Hz}$ rotation speed and between $0.2 \mathrm{~cm}$ and $2 \mathrm{~cm}$ for the $5 \mathrm{~Hz}$ rotation speed. The second scaling range occurs at scales 
below $0.8 \mathrm{~cm}$ and below $0.2 \mathrm{~cm}$ for the two rotation speeds, respectively. The exponents extracted from the large-scale end are shown in the insets of fig. 2(b) and (d). Note that the variation of the exponents vs. $n$ follows a straight line up to order 6 . This line is in excellent agreement with the Kolmogorov prediction $n / 3$ with no noticeable deviation and is therefore consistent with the measured value of the spectral exponent $\alpha$ for the surfactant solution which is very close to $5 / 3$. The absence of a deviation from the $n / 3$ law seems to indicate an absence of intermittency for this range of scales. This is the first surprising result as it shows that despite the complexity of the fluid used, a major feature of turbulence phenomenology, i.e., intermittency, is basically absent. On the other hand, the exponents extracted from the small-scale range increase at first but beyond $n=3$, they no longer vary; they even show a tendency towards a saturation or a slight decrease (see insets of fig. 1(d) and (f)). This behavior, as far as we know, has not been reported before and seems at odds with the variation of the power spectrum which shows no distinction between the small-scale range and the large-scale range (delimited by an arrow in fig. 2(a) through (c)). This variation of the exponents vs. order would indicate that the phenomenon of intermittency for the velocity fluctuations at these scales is here at its maximum and at odds with the large scales which show none. Few cases show a saturation of the exponents: one-dimensional compressible turbulence [12], granular turbulence [13], and thermal convection [14], have been shown to display such a saturation. In all these cases, the presence of strong structures (shocks in the first case and in the second case and well-defined thermal plumes in the third case) introduce large velocity gradients or large temperature gradients, respectively, giving rise to a flattening of the exponents of the structure functions. For the surfactant solution this may also be the case. It has been shown before that this solution may show the formation of gel-like patches, i.e., very viscous patches, within the solution in regions subjected to strong shear [10]. We speculate that since the turbulence engenders large shears and large shear fluctuations, the solution may actually form such patches in different places and of different sizes. If such a patch is formed, relative velocities within the patch would be very small while the patch itself would move freely in the solution. Under such conditions, the velocity gradients may be very large at the frontier between the patch and the fluid around it. We noted, for example, that the small-scale end starts at $0.8 \mathrm{~cm}$ for the lower rotation speed, while it starts at $0.2 \mathrm{~cm}$ for the higher rotation speed perhaps indicating that larger rotations give rise to smaller patches. If we estimate the deformation rate at these scales using $S_{1}(r) / r$ we find 25 and $85 \mathrm{~s}^{-1}$ which is close to the shear rates for which shear thickening occurs [10]. Since at scales below the transition, the deformation rates are higher, it is plausible that the viscosity at these small scales is much enhanced. If this is indeed the case, the enhancement of the viscosity
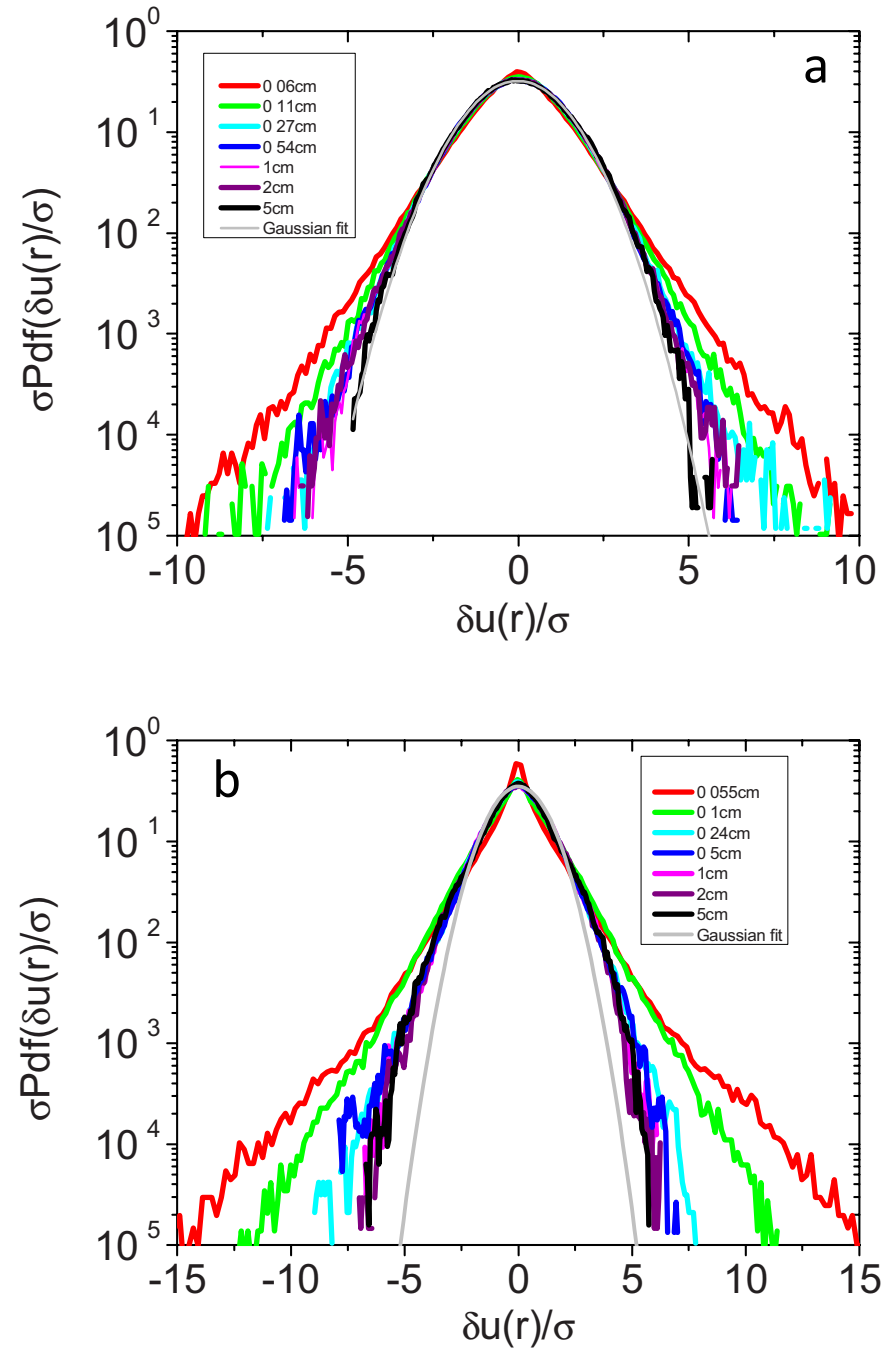

Fig. 3: (Colour on-line) Pdfs of velocity differences at different scales: (a) water, (b) surfactant solution.

will also lead to an increase of the dissipative scale which would be close to 600 micrometers. It is then possible that the small-scale range is also influenced by the dissipation within the gel patches at small scales. The possibility that the viscosity increases with the decrease in scale may also lead to modifications of how energy is transferred and dissipated.

In order to further probe the transition between the large scales, which show no intermittency, and the small scales, which show strong intermittency, we have examined the probability density functions (pdfs) of velocity differences across the scales, shown in fig. 3(a) and (b) for water and the surfactant solution, respectively. These pdfs are presented in a normalized representation: the horizontal axis has been normalized by the variance of the distribution, and the pdf normalized in such a way that the integral of the function is 1 . In such a representation, and for the case where intermittency is absent, the pdfs for the different scales should collapse onto a single curve. This is the case for neither water nor the surfactant solution at 
small scales whose pdfs display exponential-like tails and therefore large deviations with respect to the mean. Moreover, differences arise between the two different systems. For the range of scales where the structure functions show Kolmogorov scaling, the collapse of the different curves seems to work better for the surfactant solution than for water despite the fact that the pdfs for the surfactant solution are not Gaussian. In addition, and for this large-scale range, the pdfs for water seem to asymptote towards a Gaussian shape at large scales, as expected from previous experiments, while those for the surfactant solution seem to remain non-Gaussian. These observations are in line with the less intermittent character of the fluctuations for the surfactant solution: the pdfs for water seem to change gradually towards a Gaussian shape, while the surfactant pdfs remain non-Gaussian with little change as the scale varies. The non-collapse of the pdfs for the surfactant solution is manifest for the small-scale range only. Despite the fact that the scales examined are well in the scaling region of the energy spectrum, the noncollapse of the pdfs and their wide tails seems to indicate near-dissipative-range-like behavior. In this respect, our data show that such abehavior is pronounced for water but only at scales near or below the range where the energy density spectrum shows a steeper decrease, i.e., in the near-dissipative range. Note, however, that for comparable scales, the pdfs for the surfactant solution show even more important tails than those for water. This behavior suggests that scales below the transition between the two ranges, despite the scaling of the spectrum, reside in a near-dissipative-like range whose signature is absent in the energy spectrum. Is the inertial range of surfactant turbulence contaminated by large fluctuations in the dissipative scale, due to enhanced viscosity at different scales and different places, giving rise to a region where strong intermittency is observed? Our observations raise a number of questions as to how dissipation occurs in complex fluid turbulence, where viscosity variations may occur at different scales, and what role the scales in the near-dissipative range play. In addition and since the proximity of the neardissipative range is thought to play a strong role in the intermittency properties of fluid turbulence $[15,16]$, the existence of such a range and its role in complex fluid turbulence may shed new light on the phenomenology of intermittency as it is strongly inhibited at the large-scale end in our case.
In conclusion, the surfactant solutions show strong intermittency at small scales with respect to the pure water case and with respect to the polymer solution. This intermittency is then absent or very small for the larger scales. The origin of the strong intermittency at small scales has been traced back to the highly non-Gaussian statistics of the velocity fluctuations which may be due to the intrinsic properties of the solution and notably to its shear thickening character leading to the presence of very viscous parcels in the fluid. These parcels may enhance the local dissipation and therefore influence the turbulence properties at the scale of the parcel and beyond. Since another complex fluid, a polymer solution, shows very similar phenomenology as the water case, we speculate that the fine tuning of fluid properties may shed new light on how intermittency occurs in fluid turbulence.

\section{REFERENCES}

[1] МсСомв W. D., The Physics of Fluid Turbulence (Oxford, Clarendon) 1990.

[2] Frisch U., Turbulence: The Legacy of A. N. Kolmogorov (Cambridge University Press, Cambridge, England) 1995.

[3] Toms B. A., Observation on the flow of linear polymer solutions through straight tubes at large Reynolds numbers, in the Proceedings of the 1st International Rheological Congress (North-Holland, Amsterdam) 1948.

[4] Lumley J. L., Annu. Rev. Fluid Mech., 1 (1969) 367.

[5] van Doorn E., White C. M. and Sreenivasan K. R., Phys. Fluids, 11 (1999) 2387.

[6] Ouellette N. T., Xu H. and Bodenschatz E., J. Fluid Mech., 629 (2009) 375.

[7] Cadot O. et al., Phys. Rev. E, 56 (1997) 427.

[8] Cadot O., Bonn D. and Doundy S., Phys. Fluids, 10 (1998) 426.

[9] Chainais P., Aubry P. and Pinton J. F., Phys. Fluids, 11 (1999) 3524

[10] Drappier J. et al., EPL, 74 (2006) 362.

[11] de la Torre A. and Burguete J., Phys. Rev., Lett., 99 (2007) 054101.

[12] Kellay H. and Rouch J., Eur. Phys. J. B, 4 (1998) 121.

[13] Kellay H., Amarouchene Y. and Boudet J. F., Phys. Fluids, 19 (2007) 078104.

[14] Celani A., Mazzino A. and Vergassola M., Phys. Fluids, 13 (2001) 2133.

[15] Chevillard L., Castaing B. and Lévêque E., Eur. Phys. J. B, 45 (2005) 561.

[16] Frisch U. and Vergassola M., EPL, 15 (1991) 439. 